\title{
1 Optimizing smartphone-based canopy 2 hemispherical photography
}

3 *Gastón Mauro Díaz ${ }^{\mathrm{a}, \mathrm{b}}$

$4 \quad{ }^{a}$ National Scientific and Technical Research Council —Argentina (CONICET); ${ }^{\text {Patagonian }}$

5 Andes Forest Research and Extension Center (CIEFAP)

6 *Corresponding author: gdiaz@ correociefap.org.ar; Department of Geomatics, CIEFAP,

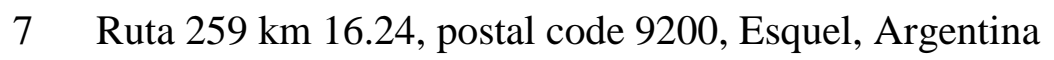

8 Running headline: Smartphone-based canopy hemispherical photography

\section{Abstract}

11 1) Hemispherical photography (HP) is a long-standing tool for forest canopy characterization. Currently, there are low-cost fisheye lenses to convert smartphones into high-portable HP equipment; however, they cannot be used whenever since HP is sensitive to illumination conditions. To obtain sound results outside diffuse light conditions, a deep-learning-based system needs to be developed. A ready-to-use alternative is the multiscale color-based binarization algorithm, but it can provide moderate-quality results only for open forests. To overcome this limitation, I propose coupling it with the model-based local thresholding algorithm. I call this coupling the

2) Methods presented here are part of the R package CAnopy IMage ANalysis (caiman), which I am developing. The accuracy assessment of the new MBCB approach was done 
with data from a pine plantation and a broadleaf native forest.

23 3) The coefficient of determination $\left(R^{2}\right)$ was greater than 0.7 , and the root mean square error (RMSE) lower than $20 \%$, both for plant area index calculation.

4) Results suggest that the new MBCB approach allows the calculation of unbiased canopy metrics from smartphone-based HP acquired in sunlight conditions, even for closed canopies. This facilitates large-scale and opportunistic sampling with hemispherical photography.

30 Key-words: image processing, canopy image, leaf area index, lens calibration

\section{Introduction}

32 Canopies are often characterized with the leaf area index (LAI), defined as one half the total

33 photosynthetic area per unit of horizontal ground surface area (Chen \& Black, 1992). Among

34 the established techniques for LAI measurement (Calders et al., 2018), hemispherical

35 photography (HP) is often chosen because of its low purchasing cost and enthusiastic

36 supporting community.

37 Each HP application requires a balance between sampling density and sample accuracy.

38 Heterogeneos canopies require large sampling efforts (Díaz et al., 2021), while monitoring

39 phenology, high accuracy (Lang et al., 2017). Other applications require opportunistic

40 sampling, common in regions with low terrestrial accessibility. However, most research faces

41 the accuracy problem and little is known about solutions for large-scale and opportunistic

42 sampling.

43 Error sources for traditional processing protocols are (1) acquiring photographs in non- 
diffuse light conditions and (2) wrong exposure settings; besides, independent from the processing protocol, there is improper lens calibration (Fournier \& Hall, 2017). These error sources are often combined when HP is used without due training. This is more common now than ever since cheap fisheye lenses are available to convert smartphones in HP equipment.

With the easily available free HP software packages, wrong results can be produced if incorrect parameters are set, such as erroneous lens parameters. Among the free software, CAN-EYE is the most complete since it solves from lens calibration to canopy indices calculation (https://www6.paca.inrae.fr/can-eye/). Other packages tend to focus on a particular link of the processing chain. For instance, CIMES can calculate a wide variety of well-documented canopy indices (Gonsamo et al., 2011), and the R package CAnopy IMage

54 ANalysis (caiman) includes original binarization algorithms (Díaz et al., 2021).

In 2016, I started the development of the 'caiman' package to distribute the multiscale colorbased binarization (MCB) algorithm (Díaz \& Lencinas, 2015). Later, I introduced the modelbased local thresholding (MBLT) algorithm (Díaz \& Lencinas, 2018) and, driven by user demands, I expanded some functionalities, such as full-frame support (Bianchi et al., 2017) and adaptive binarization (Martin et al., 2020). Altrought the first proof of concept suggesting

60 that the package can process smartphone-based HP was already published (Bianchi et al.,

61 2017), I have refined important details so a document accounting for those changes is needed.

62 Also, I believe that the calibration of fisheye lenses for smartphones has been overlooked

63 since they tend to strongly deviate from the standard projections. Here I contribute to 64 amending that. The MCB algorithm was developed to process photographs acquired in sunny days (sunlight 66 images) — a particular non-diffuse light condition — and to mitigate the blooming effect, 
linked to incorrect exposure settings (Díaz \& Lencinas, 2015). However, a recent evaluation closed canopies (Díaz et al., 2021). Till now, the deep-learning (DL) approach by Díaz et al. (2021) is the only available solution for processing sunlight images with sound accuracy. But, its implementation is time-consuming and requires DL know-how.

Here, I propose to couple the MBLT and MCB algorithms (hereafter, MBCB approach). This new workflow is computational demanding but here I show that it has the potential to provide unbiased results for both broadleaf and coniferous woods, requiring only basic knowledge of $\mathrm{R}$ scripting. The purpose of this paper is twofold: to present the MCBC approach and to evaluate it with a dataset involving both smartphone-based and regular HP.

\section{Lens calibration}

78 Canopy hemispherical photographs are mostly taken pointing to the zenith. Given that, the vertical line at the lens location is the optical axis. The angle between the optical axis and any other line passing through the lens is the zenith angle $(\theta)$. As isolines in a map, points in the image with the same $\theta$ form a circle; therefore, each $\theta$ has a corresponding radius. The radius expressed as a fraction of the radius for $90^{\circ} \theta$ is the relative radius $(R)$. Following a standard method, the 'caiman' package uses a polynomial curve to model the relation between $\theta$ and

$84 R$. The fitted model is called a lens projection function.

Based on Clark \& Follin (1988), I developed a method to estimate projection coefficients that only requires inexpensive everyday materials: printing an A1 size PDF provided along with

87 the package, a tripod, a table, a standard yoga mat, and push pins of different colors. I programmed the calibrate_lens() function for the required calculations. Fig. 1 shows the FC- 
90 about the latter can be found in Díaz \& Lencinas (2018) S3. Also, Fig. 1 shows the projection

91 function of fisheye lenses for smartphones that I found in the Argentinian market.

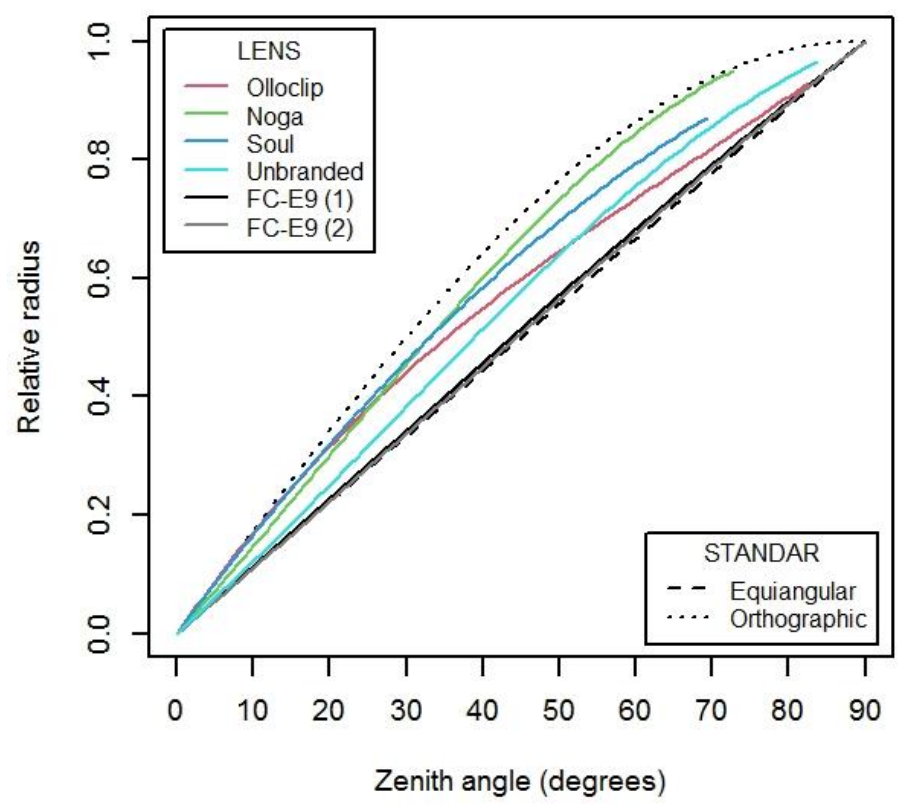

94 Fig. 1. Lens projection functions of one smartphone lens with long-term market presence (Olloclip), three generic smartphone lenses (Noga, Soul, and Unbranded), and two versions for the well-established FC-E9 lens:

96 (1) validated, and (2) adjusted with the method presented here. For comparison, two standard projections.

99 Fig. 2 helps understanding the different kinds of photographs that can be acquired and what is

100 the best processing alternative for them. If sampling with high accuracy is needed, then

101 diffuse-light images acquired with professional equipment should be used. For smartphone-

102 based HP (i.e., for non-professional equipment), the acquisition of diffuse-light images can 

rigorous systematic sampling.

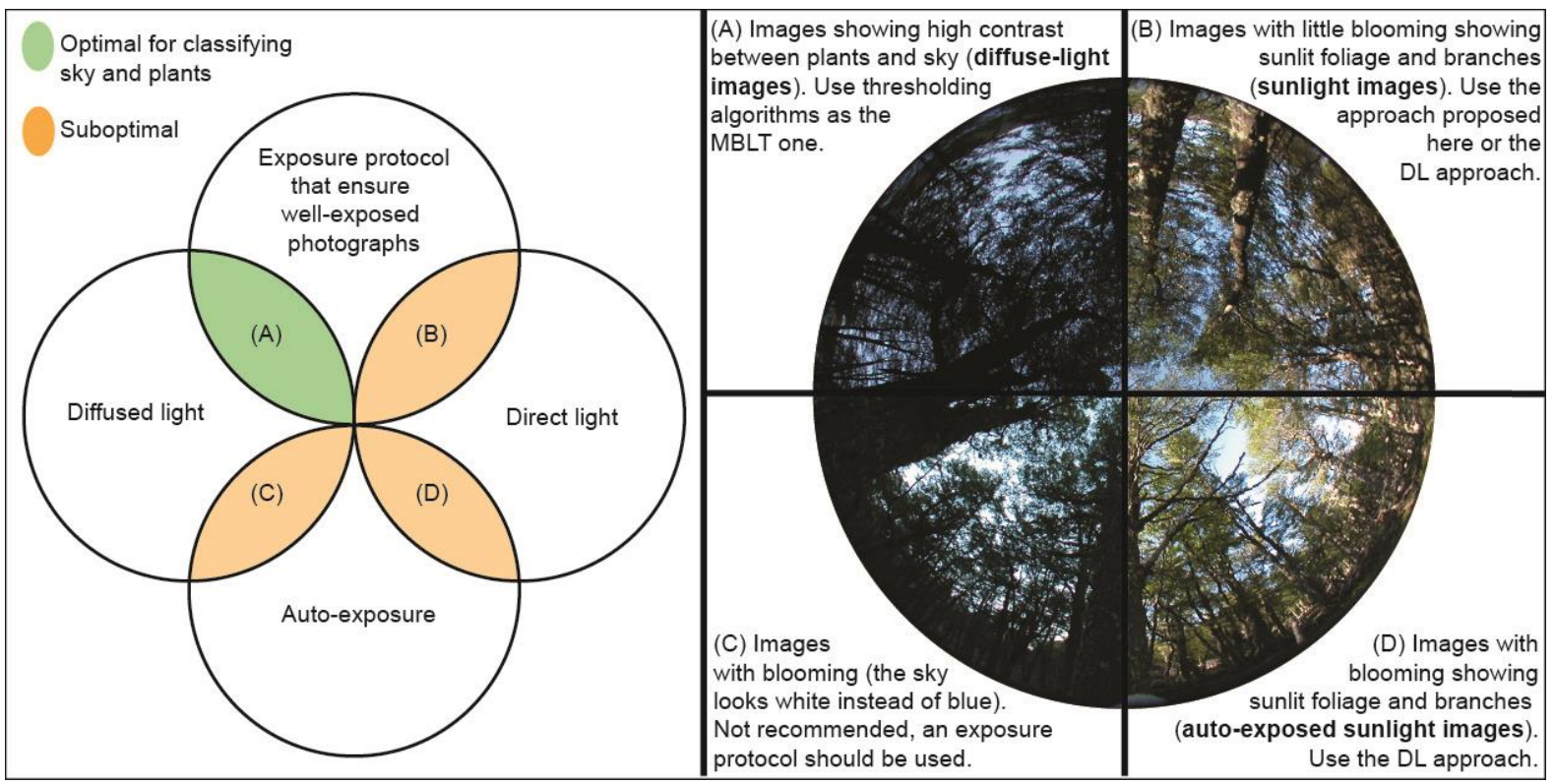

112 Fig. 2. Relation between camera exposure and light conditions. (C) and (D) are not observed in open canopies

113 because the auto-exposure method provides a result similar to an exposure protocol. The photographs were

114 taken in a Nothofagus pumilio forest with a FC-E9 lens (see Fig. 1). MBLT stands for model-based local

115 thresholding, MCB for multiscale color-based binarization, and DL for deep learning. 


\section{Evaluation of the MBCB approach}

\section{Methods}

119 Two pieces of equipment were used to acquire the hemispherical photographs; a Nikon FC-

120 E9 fisheye converter attached to a Nikon Coolpix 5700 camera, which produces circular

121 fisheye photographs with 1490 pixel diameter at $180^{\circ}$ field of view (FOV) - equivalent to

122745 pixel radius for $90^{\circ} \theta-$; and a Olloclip lens attached to an iPhone 6 Plus, which

123 produces an incomplete circular image (Fig. 3) with $165^{\circ}$ diagonal FOV, $160^{\circ}$ horizontal

$124 \mathrm{FOV}$, and $110^{\circ}$ vertical FOV. Its lens projection is $R=1.06065 \theta-0.49054 \theta^{2}+$

$1250.14044 \theta^{3}$ (Fig. 1). The extrapolated radius for $90^{\circ} \theta$ is 1786 pixels. That is 2.4 times

126 greater than the FC-E9 radius; however, since strong blurring occurs in the Olloclip lens after

$12755^{\circ} \theta$, more pixels is not equivalent to more sharpness. 


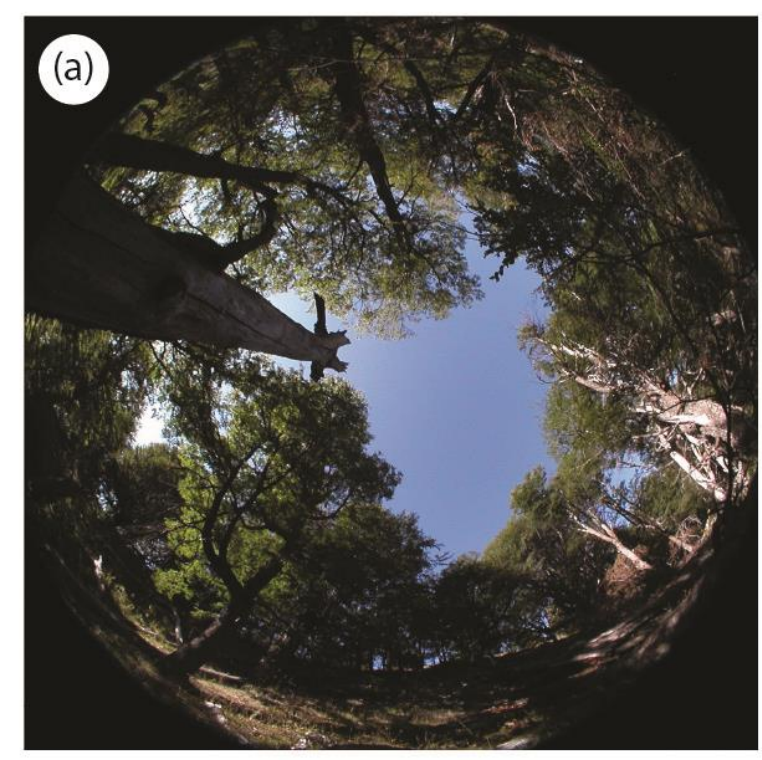

(b)
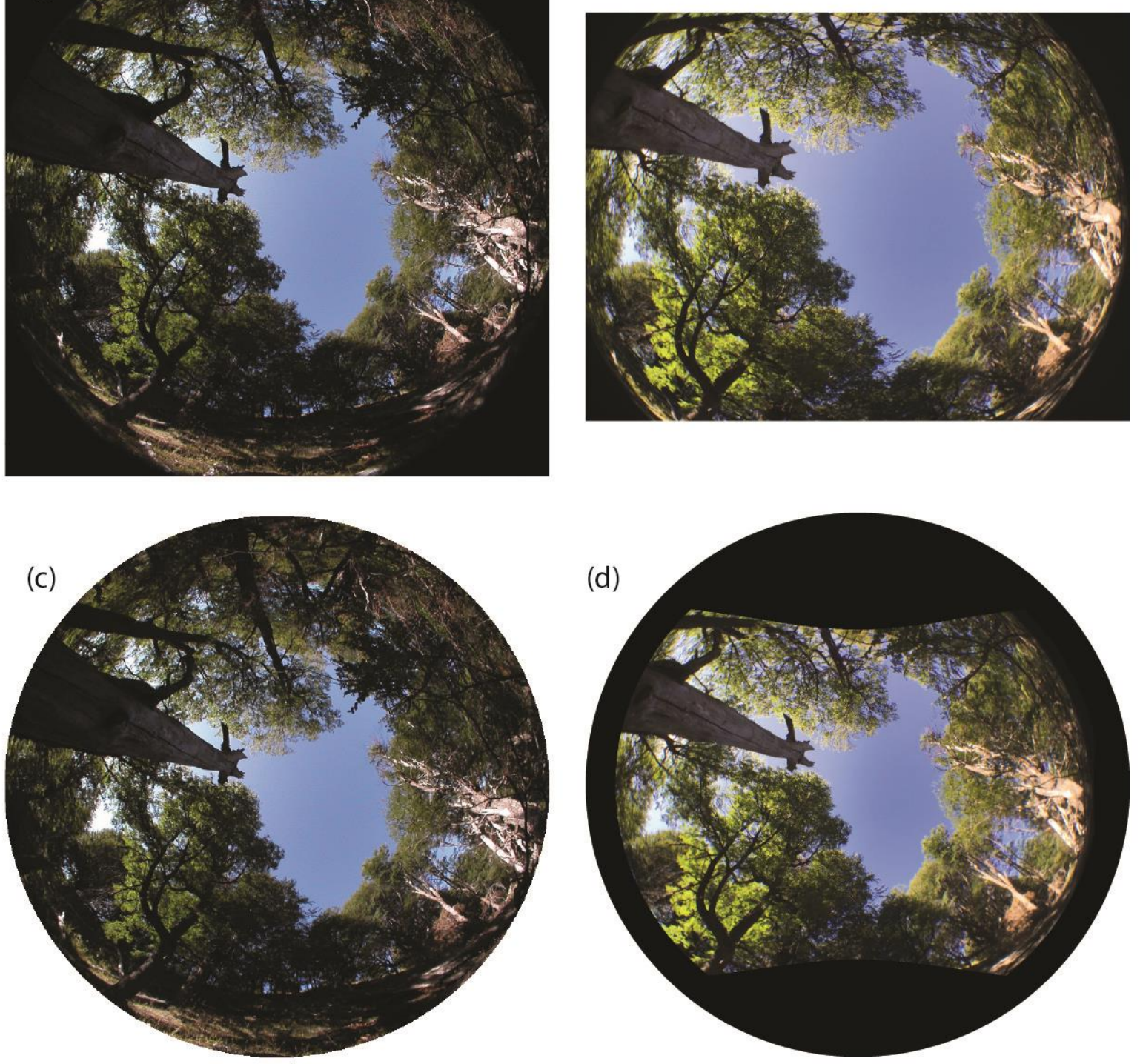

130 Fig. 3. Original — (a) and (b) — and reprojected —(c) and (d) — canopy hemispherical photographs of a Pinus

131 ponderosa plantation. Both reprojected to the equiangular projection. (a) and (c) taken with the FC-E9 lens; (b)

132 and (d), with the Olloclip lens.

134 Two datasets were used; the one described in Díaz et al. (2021) section 2.1.3, from a native

135 Nothofagus pumilio forest (broadleaf species); and another presented here for the first time, from a Pinus ponderosa plantation. 
137 The broadleaf forest dataset was acquired in February 2020, in the Huemules Norte

138 experimental unit (42 $\left.46^{\prime} 47^{\prime \prime} \mathrm{S}, 7^{\circ} 27^{\prime} 53^{\prime \prime} \mathrm{W}\right)$. On 104 locations (photosites), two sunlight

139 photograph was taken, one with the FC-E9 lens and another with the Olloclip. To calculate

140 reference values, we used the DL system from Díaz et al. (2021) and the sunlight FC-E9

141 images to obtain plant area index (PAI) and effective PAI (PAIe), two canopy indices

142 strongly related to LAI.

143 New data were acquired in a pine plantation near the city of Esquel, Argentina (42 $53^{\prime} 49.4^{\prime \prime}$

$144 \mathrm{~S}, 71^{\circ} 21^{\prime} 36.5^{\prime \prime} \mathrm{W}$ ), on September 9th, 2020. At dawn, diffuse-light images (FC-E9 lens) were

145 acquired following the same protocols used by Díaz \& Lencinas (2018). Fithteen diffuse-light

146 images were acquired along a transec selected to maximize canopy openness variability. Each

147 photosite was flagged. Sunlight images (FC-E9 and Olloclip lenses) were acquired in those

148 photosites in the morning (one missing photo), midday, and afternoon. Sun elevation was $25^{\circ}$,

$14941^{\circ}$, and $36^{\circ}$, respectively. All of these photographs were taken at $1.25 \mathrm{~m}$ from the floor. To

150 calculate the reference values, I binarized the diffuse-light images with the MBLT algorithm

151 and ingested them in the Hemisfer 3.1 software package

152 (https://www.schleppi.ch/patrick/hemisfer). Only the range from $20^{\circ}$ to $70^{\circ} \theta$ was used in the

153 calculations. See Díaz \& Lencinas (2018) for more methodological details.

154 The Olloclip images were acquired with auto-exposure, except when abundant saturation and

155 blowing were observed on the smartphone screen. Then, the exposure was manipulated to

156 diminish it. The FC-E9 sunlight images were always acquired in RAW format and

157 underexposing 1 to 2 stops relative to the on-site auto-exposure, following Beckschäfer et al.

158 (2013). The RAW files were converted into 16-bit TIFFs with the ViewNX-i software by

159 Nikon. For testing, three image versions were produced by modifying the exposure

160 compensation parameter from 0 (default) to 1 and -1. 
161 Sunlight images were binarized with the MBCB approach. Since this article aims at researchers not specialized in HP, the general-purpose binarization algorithm by Ridler \&

163 Calvard (1978), known as ISODATA, was also used to serve as a common baseline. From

164 the binarized sunlight images, PAIe and PAI were calculated with Hemisfer as done for the

165 binarized diffuse-light images.

\section{Results and discussion}

167 The pine plantation showed maximum reference values lower than the broadleaf forest (1.2 to

168 2.8 PAIe and 3.3 to 5.6 PAI vs. 1.1 to 4.9 PAIe and 2.9 and 7.3 PAI). Sunlight images from

169 the former allowed accurate PAIe and PAI calculation, but for the latter, only accurate PAIe

170 (Table1). As expected, the baseline performed worse than the MBCB approach, being that

171 more evident for the broadleaf forest (Table 1). However, although biased, the baseline

172 showed a good correlation with the reference values for pine PAIe. To interpret this, it is

173 necessary to consider that pine photographs were acquired under a complete clear sky, while

174 broadleaf photographs in days with broken clouds in the morning. Therefore, it is uncertain if

175 the type of canopy or the sky conditions affected these correlations. However, based on Fig.

$1764 \mathrm{~b}$ (see next paragraph), seems to be the former.

177 Difference in the solar disk position introduced a random error (Fig. 4a), so repeated

178 measurement with hours of difference could increase accuracy. The source of random error is

179 probably related to variations in the amount of sunlit canopy depicted in the photographs,

180 which depends on the solar disk position and the canopy itself. This could explain the

181 absence of an optimal solar elevation. The lack of difference between morning and afternoon

182 in Fig. 4b might suggest that broken clouds, only presented in the morning, did not affect the

183 results, but further research is needed. 
184 Table 1. Accuracy assessment of both plant area index (PAI) and effective PAI (PAIe) calculated from sunlight

185 hemispherical photographs binarized with the new workflow (MBCB approach) and a baseline (ISODATA

186 algorithm). RMSE stands for root mean square error, $R^{2}$ for coefficient of determination and EC for the

187 exposure compensation parameter used to develop the RAW files. Bold type indicates best performing.

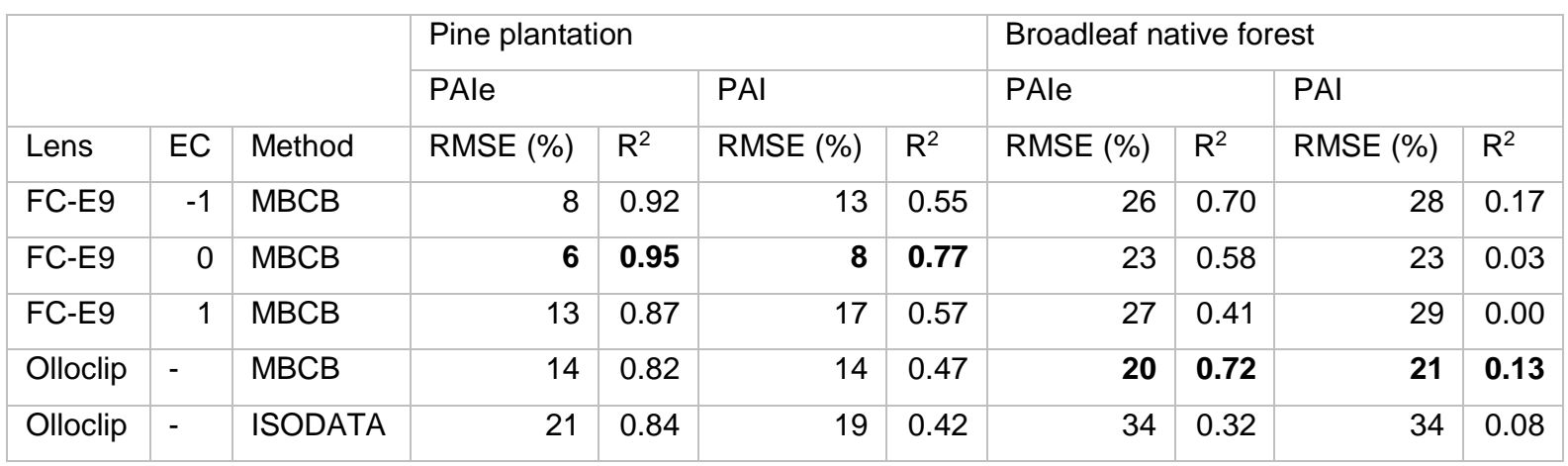

188

189

(a) Pine plantation

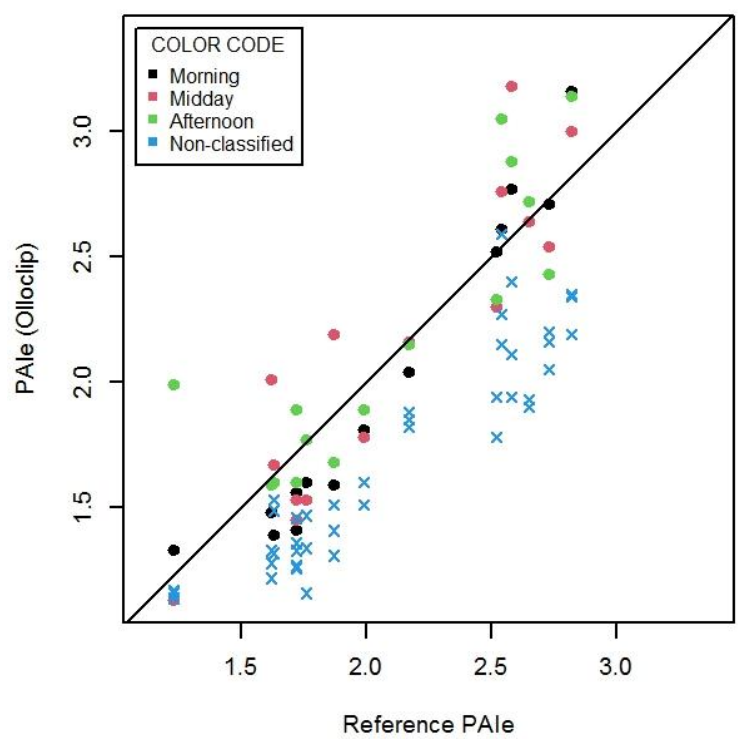

(b) Broadleaf forest

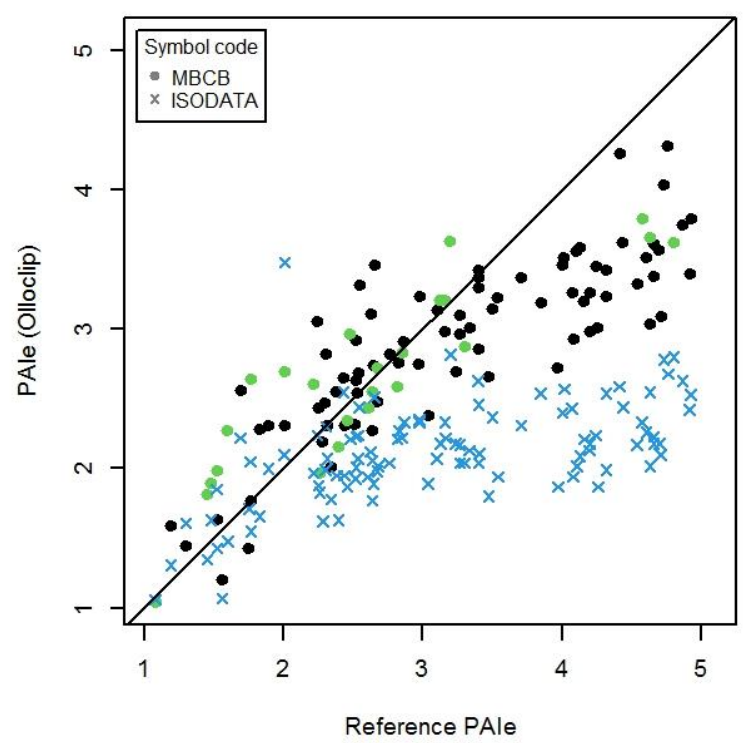

190

191 Fig. 4. Accuracy assessment of smartphone-based hemispherical photography in sunlight conditions. PAIe

192 stands for effective plant area index, MBCB for the new approach presented here, and ISODATA is the

193 baseline. Solid lines are the 1:1 relationship. 
194 Varying the EC parameter provided insight for the development of exposure protocols and

195 the potential of the MBCB approach. Based on the calculated errors, I selected the best

196 version of each image and visually analyzed them. It seems that a clear sky depicted in light

197 blue (as it is shown in Fig. 3) should be pursued during photograph acquisition. This protocol

198 is easy to implement with smartphone-based equipment. Regarding the potential of the

199 MBCB approach for supporting PAIe calculation, $R^{2}$ and RMSE for the best version of each

200 broadleaf image were 0.87 and $14 \%$, respectively. And, no bias was observed in the higher

201 values.

\section{The future of the 'caiman' package}

203 The package is evolving as more research is done and users provide feedback. This paper

204 might lead to many new users and a consequent consolidation of its main features. After that,

205 my goal is to conduct a complete reengineering of the package to improve its usability, polish

206 the code, rename it as 'rcaiman', and submit it to the main repository for R packages, the

207 Comprehensive R Archive Network (CRAN).

\section{Conclusion}

209 Smartphone cameras, besides its low-cost and universality, are not inherently superior to

210 other digital cameras used for HP. The same error sources valid for digital HP are valid for

211 smartphone-based HP. Acquisitions in sunlight conditions are common in large-scale and

212 opportunistic sampling, which benefit from the portability of smartphones. I recommend

213 manipulating the exposure to produce sunlight images with the clear sky depicted in light

214 blue. A ready-to-use solution for binarizing sunlight images was presented, which I call the

215 MBCB approach. Although further evaluation is recommended, results suggest that this new

216 solution allows the calculation of unbiased PAIe with smartphone-based HP acquired in

217 sunlight conditions. 


\section{Acknowledgements}

219 I thank Veronica Verano for language help.

\section{Data accessibility}

221 The 'caiman' package is available in a GitHub repository

222 (https://github.com/GastonMauroDiaz/caiman).

223 The new workflow (the MBCB approach) is available as a script in a Gist repository

224 (https://gist.github.com/GastonMauroDiaz/1b5962c7e4c2ccebe4c6e4223a19fba1).

225 Data are available in an OSF repository (https://osf.io/atywm/)

\section{References}

227 Beckschäfer, P., Seidel, D., Kleinn, C., \& Xu, J. (2013). On the exposure of hemispherical

228 photographs in forests. iForest - Biogeosciences and Forestry, 6(4), 228-237.

229 https://doi.org/10.3832/ifor0957-006

230 Bianchi, S., Cahalan, C., Hale, S., \& Gibbons, J. M. (2017). Rapid assessment of forest

231 canopy and light regime using smartphone hemispherical photography. Ecology and

232 Evolution, 7(24), 10556-10566. https://doi.org/https://doi.org/10.1002/ece3.3567

233 Calders, K., Origo, N., Disney, M., Nightingale, J., Woodgate, W., Armston, J., \& Lewis, P. 234 (2018). Variability and bias in active and passive ground-based measurements of effective 235 plant, wood and leaf area index. Agricultural and Forest Meteorology, 252, 231-240.

236 https://doi.org/https://doi.org/10.1016/j.agrformet.2018.01.029

237 Chen, J. M., \& Black, T. A. (1992). Defining leaf area index for non-flat leaves. Plant, Cell 238 \& Environment, 15(4), 421-429. https://doi.org/10.1111/j.1365-3040.1992.tb00992.x 
239 Clark, J. A., \& Follin, G. M. (1988). A simple "equal area" calibration for fisheye

240 photography. Agricultural and Forest Meteorology, 44(1), 19-25.

241 https://doi.org/https://doi.org/10.1016/0168-1923(88)90030-5

242 Díaz, G. M., \& Lencinas, J. D. (2018). Model-based local thresholding for canopy

243 hemispherical photography. Canadian Journal of Forest Research, 48(10), 1204-1216.

244 https://doi.org/10.1139/cjfr-2018-0006

245 Díaz, G. M., \& Lencinas, J. D. (2015). Enhanced gap fraction extraction from hemispherical

246 photography. IEEE Geoscience and Remote Sensing Letters, 12(8), 1785-1789.

247 https://doi.org/10.1109/LGRS.2015.2425931

248 Díaz, G. M., Negri, P. A., \& Lencinas, J. D. (2021). Toward making canopy hemispherical

249 photography independent of illumination conditions: a deep-learning-based approach.

250 Agricultural and Forest Meteorology, 296, 108234.

251 https://doi.org/https://doi.org/10.1016/j.agrformet.2020.108234

252 Fournier, R. A., \& Hall, R. J. (Eds.). (2017). Hemispherical photography in forest science:

253 Theory, methods, applications. Springer Netherlands. https://doi.org/10.1007/978-94-024-

$254 \quad 1098-3$

255 Gonsamo, A., Walter, J.-M. N., \& Pellikka, P. (2011). CIMES: A package of programs for

256 determining canopy geometry and solar radiation regimes through hemispherical

257 photographs. Computers and Electronics in Agriculture, 79(2), 207-215.

258 https://doi.org/https://doi.org/10.1016/j.compag.2011.10.001

259 Kuusk, A., \& Paas, M. (2007). Radiometric correction of hemispherical images. ISPRS

260 Journal of Photogrammetry and Remote Sensing, 61(6), 405-413. 
261 https://doi.org/https://doi.org/10.1016/j.isprsjprs.2006.10.005

262 Lang, M., Nilson, T., Kuusk, A., Pisek, J., Korhonen, L., \& Uri, V. (2017). Digital

263 photography for tracking the phenology of an evergreen conifer stand. Agricultural and

264 Forest Meteorology, 246, 15-21.

265 https://doi.org/https://doi.org/10.1016/j.agrformet.2017.05.021

266 Martin, D. A., Wurz, A., Osen, K., Grass, I., Hölscher, D., Rabemanantsoa, T., Tscharntke,

267 T., \& Kreft, H. (2020). Shade-tree rehabilitation in vanilla agroforests is yield neutral and

268 may translate into landscape-scale canopy cover gains. Ecosystems.

269 https://doi.org/10.1007/s10021-020-00586-5

270 Ridler, T., \& Calvard, S. (1978). Picture thresholding using an iterative selection method.

271 IEEE Transactions on Systems, Man, and Cybernetics, 8(8), 630-632.

272 https://doi.org/10.1109/tsmc.1978.4310039 